\title{
Anti-Integrins for the Treatment of Inflammatory Bowel Disease: Current Evidence and Perspectives
}

\author{
John Gubatan (1D' \\ Kian Keyashian ' \\ Samuel JS Rubin (iD) ${ }^{2,3}$ \\ Jenny Wang ${ }^{2}$ \\ Cyrus A Buckman (D) ${ }^{2}$ \\ Sidhartha Sinha' \\ 'Division of Gastroenterology and \\ Hepatology, Stanford University School \\ of Medicine, Stanford, CA, USA; \\ ${ }^{2}$ Stanford University School of Medicine, \\ Stanford, CA, USA; ${ }^{3}$ Immunology \\ Program, Stanford University School of \\ Medicine, Stanford, CA, USA
}

\begin{abstract}
Leukocyte trafficking to the gastrointestinal tract is recognized to play a role in the pathogenesis of inflammatory bowel disease (IBD). Integrins are expressed on immune cells and interact with cell adhesion molecules (CAM) to mediate leukocyte trafficking. Blockade of the gut-tropic integrin $\alpha 4 \beta 7$ and its subunits has been exploited as a therapeutic target in IBD. Natalizumab (anti- $\alpha 4$ ) is approved for moderate to severe Crohn's disease (CD), but its use is limited due to potential risk of progressive multifocal leukoencephalopathy. Vedolizumab (anti- $\alpha 4 \beta 7$ ) is approved for the treatment of ulcerative colitis (UC) and CD. It is the most widely used anti-integrin therapy in IBD and has been shown to be effective in both induction and maintenance therapy, with a favorable safety profile. Several models incorporating clinical, genetic, immune, gut microbial, and vitamin D markers to predict response to vedolizumab in IBD have been developed. Etrolizumab (anti- $\beta 7$ ) blocks leukocyte trafficking via $\alpha 4 \beta 7$ and cell adhesion via $\alpha E \beta 7$ integrins. Large phase 3 clinical trials evaluating efficacy of etrolizumab in the induction and maintenance of patients with IBD are underway. Other investigational anti-integrin therapies include abrilumab (anti- $\alpha 4 \beta 7$ IgG2), PN-943 (orally administered and gut-restricted $\alpha 4 \beta 7$ antagonist peptide), AJM300 (orally active small molecule inhibitor of $\alpha 4$ ), and ontamalimab (anti-MAdCAM-1 IgG).
\end{abstract}

Keywords: anti-integrin, inflammatory bowel disease, ulcerative colitis, Crohn's disease, vedolizumab, natalizumab, etrolizumab

\section{Introduction}

Inflammatory bowel disease (IBD), which includes ulcerative colitis (UC) and Crohn's disease (CD), is a chronic inflammatory disorder of the gastrointestinal tract. The etiology of IBD is unclear, but the current thought is that genetic and environmental factors lead to immune dysregulation, intestinal barrier dysfunction, and loss of tolerance to commensal gut bacteria. ${ }^{1,2}$ The incidence of IBD is increasing worldwide and associated with increased healthcare utilization and poor quality of life. ${ }^{3,4}$ The management of IBD often involves immunosuppressive therapies such as corticosteroids, immunomodulators, small molecules, and biologic agents that inhibit proinflammatory cytokine pathways (eg, anti-TNF- $\alpha$, anti-p40 subunit of IL-12/23). ${ }^{5}$ More specific therapies that target gut-tropic integrins and block leukocyte trafficking to the gastrointestinal tract provide an alternative therapeutic approach to systematic immunosuppression in IBD. In this review, we explore the role of integrins in leukocyte trafficking to the gut and highlight currently approved and emerging anti-integrin therapies in IBD (summarized in Figure 1). 


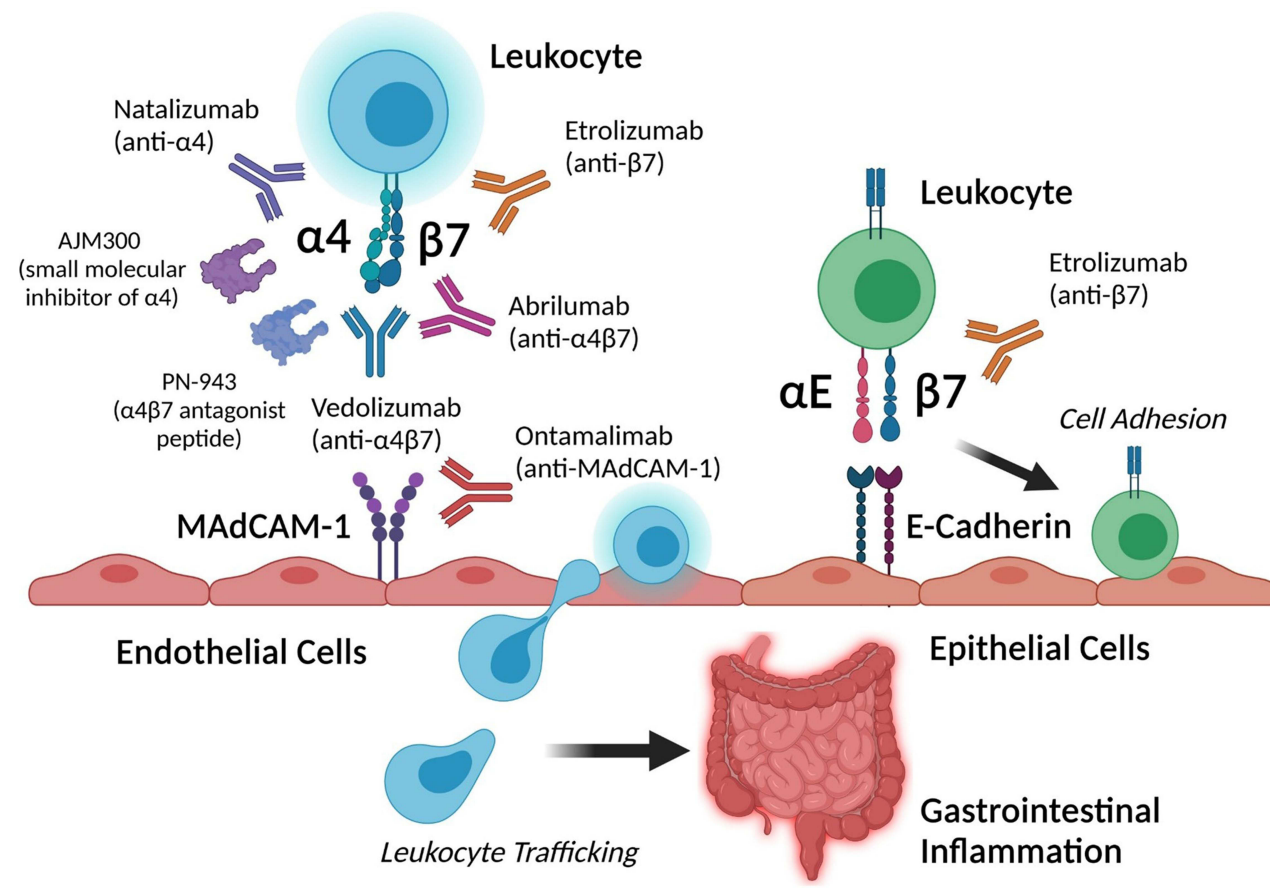

Figure I Summary of current and investigational anti-integrin therapies and their targets in inflammatory bowel disease. Created with BioRender.com.

\section{Role of Integrins in Leukocyte Trafficking to Gastrointestinal Tract and Pathogenesis of Inflammatory Bowel Disease}

Integrins are cell surface transmembrane glycoproteins that mediate cell-cell interactions and play critical roles in immune cell proliferation, signaling, and trafficking. ${ }^{6,7}$ Integrins consist of heterodimers involving $\alpha$ and $\beta$ subunits that bind to cell adhesion molecules (CAM) on other cell types or to the extracellular matrix. ${ }^{8}$ Immune cells express integrins $^{9}$ that bind to tissue-specific CAM which mediates their trafficking from circulation or lymphoid tissue to specific peripheral tissue including the gastrointestinal tract (gutassociated lymphoid tissue, GALT). ${ }^{9}$ Several integrins have been characterized to confer specific trafficking to the gut ("gut-tropic integrins"). The integrin $\alpha \mathrm{L} \beta 2$ (LFA-1) is expressed by lymphocytes and natural killer (NK) cells and interacts with intercellular adhesion molecules (ICAMs) to mediate lymphocyte trafficking to mesenteric lymph nodes. ${ }^{10}$ The integrin $\alpha 4 \beta 1$ (VLA-4) is expressed on most leukocytes and neutrophils and interacts with vascular cell adhesion molecule 1 (VCAM-1). The $\alpha 4 \beta 1$ integrin plays a role in the homing of memory and effector $\mathrm{T}$ lymphocytes to the brain as well as to inflamed intestinal tissue. ${ }^{11}$ The $\alpha 4 \beta 7$ integrin heterodimer of memory $\mathrm{T}$ and $\mathrm{B}$ cells is a cell- surface glycoprotein that is critical for leukocyte homing in the gut. The $\alpha 4 \beta 7$ integrin interacts with mucosal addressincell adhesion molecule 1 (MAdCAM-1) expressed on high endothelial venules in GALT, which is expressed on the intestinal vascular endothelium; ${ }^{12}$ this interaction plays an important role in immune surveillance of the gastrointestinal tract and the gut-selective nature of lymphocyte trafficking as it enables lymphocytes to travel across the vascular endothelial barrier to GALT or intestinal lamina propria. ${ }^{13}$ The $\alpha \mathrm{E}$ integrin (also known as CD103) can dimerize with the $\beta 7$ integrin to form $\alpha \mathrm{E} \beta 7$ which binds to its ligand E-cadherin. $\alpha \mathrm{E} \beta 7$ expression has been demonstrated on various immune cells including intraepithelial $\mathrm{T}$ lymphocytes and dendritic cells (DCs). ${ }^{14,15} \alpha \mathrm{E} \beta 7$ expression on intraepithelial lymphocytes enables their retention on the epithelial layer of the intestinal tract. ${ }^{16}$

Since integrins mediate trafficking and retention of immune cells to the gastrointestinal tract, it is not surprising that they are implicated in the pathogenesis of IBD. A prior genome-wide association study demonstrated that IBD was associated with immune activation of multiple integrin genes (ITGA4, ITGB8, ITGAL, ICAM1). These four loci were also associated with risk of IBD. ${ }^{17}$ Patients with IBD have been shown to have upregulated gut-tropic integrins and cell adhesion molecules in inflamed intestinal tissue. For example, circulating $\alpha 4 \beta 7+\mathrm{CD} 4 \mathrm{~T}$ cells are 
increased among patients with $\mathrm{UC}$ and $\mathrm{CD} .{ }^{18,19}$ Likewise, VCAM-1 and MAdCAM-1 are upregulated in IBD. ${ }^{20}$ These early observations provided a rationale for blockade of integrins or cell adhesion molecules as a therapeutic target in IBD. In early preclinical studies, blockade with either anti- $\beta 7$ or anti-MAdCAM-1 monoclonal antibodies significantly reduced the severity of colitis in murine $T$ cell transfer models of IBD. ${ }^{21}$ Similarly, antibody blockade of $\alpha 4$ or $\alpha 4 \beta 7$ improved histologic inflammation in a cottontop tamarin monkey colitis model. ${ }^{22}$ The remainder of the review will focus on human studies summarizing current evidence of anti- $\alpha 4$ (natalizumab), anti- $\alpha 4 \beta 7$ (vedolizumab), anti- $\beta 7$ (etrolizumab), and other emerging antiintegrin therapies for the management of IBD.

\section{Anti-Integrin Therapy: Natalizumab (Anti-a4)}

Natalizumab (Tysabri, Elan Pharmaceuticals, and Biogen Idec) is a humanized IgG4 monoclonal antibody against the $\alpha 4$ subunit of the integrin heterodimer, effectively targeting $\alpha 4 \beta 1$ and $\alpha 4 \beta 7$, thereby inhibiting leukocyte adhesion and migration from the vasculature into tissue. ${ }^{23}$ Initially FDA-approved for multiple sclerosis, natalizumab was also shown to be potentially clinically effective and safe in moderate to severe CD during early phase studies. ${ }^{24,25}$ Subsequent FDA approval for moderate to severe CD was based on ENACT-1 and ENACT-2, two randomized, double-blind, placebo-controlled trials conducted at 142 centers between 12/2001 and 3/2004. ${ }^{26}$ ENACT-1, which enrolled 905 patients with moderate to severe CD, demonstrated 10-week CDAI-based clinical response for natalizumab to be $56 \%$ versus $49 \%$ for placebo $(P=0.05)$. The subsequent ENACT-2, which enrolled 339 patients that were responsive to natalizumab from ENACT-1, showed a statistically significant sustained response through week $36(61 \%$ versus $28 \%, P<0.001)$ and sustained remission ( $44 \%$ versus $26 \%, P=0.003$ ); $54 \%$ with initial response continued to respond at week 60. After approval, real-world data from tertiary referral centers confirmed clinical response of up to $60 \%$ at 1 year, with particular benefit in non-penetrating $\mathrm{CD} .{ }^{27-29}$

In 2005, case reports surfaced of the association between natalizumab and progressive multifocal leukoencephalopathy (PML) in 2 patients with multiple sclerosis and 1 patient with $\mathrm{CD} .{ }^{30,31}$ The disorder resulting in lytic demyelination in the central nervous system, with known pathogenetic association with the JC virus, results in up to a $25 \%$ mortality and permanent neurologic deficits. ${ }^{32}$ In the initial case report in CD, JC virus DNA was first noted 3 months after starting natalizumab, with dramatic increase subsequently. ${ }^{31}$ In the larger IBD population studied, tertiary center data showed seropositivity for JC virus to be above $65 \%$ in those tested, with risk factors including previous thiopurine use. Seroconversion was also shown to occur at $4.5 \%$ after 22 months of treatment. ${ }^{33}$

Initial risk stratification algorithms revealed higher risk of PML with JC virus antibody positivity, use of immunosuppression, and longer treatment duration beyond 2 years. More recently, patient-level data from 4 large open-label studies of $>37,000$ natalizumab-treated patients showed the point incidence of PML to be $0.4 \%$, with $99 \%$ of patients testing positive for JC virus 6 months before the diagnosis of PML. ${ }^{34}$ This study also showed that with JC virus antibody negativity, $<0.07 / 1000$ patients develop PML. In those with JC virus antibody positivity, 1.7\% develop PML over 6 years if not on additional immunosuppression, with rates of $2.7 \%$ if concomitant immunosuppression was used. This association led to suspension of the drug for clinical use in 2005, with subsequent reintroduction through the TOUCH program (Tysabri Outreach: Unified Commitment to Health). Given safety concerns, the place for natalizumab in management of moderate to severe CD seems limited. Natalizumab is currently not approved for IBD in Europe. ${ }^{35}$

\section{Anti-Integrin Therapy: Vedolizumab

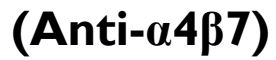

Vedolizumab (Takeda) is a humanized monoclonal IgG1 antibody that targets the $\alpha 4 \beta 7$ heterodimer. The antibody selectively blocks $\alpha 4 \beta 7$ :MAdCAM-1 interactions - preventing leukocyte adhesion to intestinal endothelium - but does not affect $\alpha 4 \beta 1$ :VCAM-1 interactions. ${ }^{36}$ Vedolizumab is FDA-approved for induction and maintenance therapy in patients with CD and CD. In GEMINI 1 (NCT00783718), two integrated randomized, double-blind, placebo-controlled trials of vedolizumab in patients with $\mathrm{UC}$ with active disease were performed. ${ }^{37}$ In the induction therapy trials, 374 patients (cohort 1) received vedolizumab or placebo intravenously at weeks 0 and 2 , and 521 patients (cohort 2) received open-label vedolizumab at weeks 0 and 2, and response (defined by a reduction in Mayo score of at least 3 points and a decrease of at least 30\% from baseline) was assessed at week 6. Response rates at week 6 were $47.1 \%$ and $25.5 \%$ among patients in the vedolizumab group and placebo group, 
respectively $(P<0.001)$. In the maintenance therapy trials, patients in either cohort who had a response to vedolizumab at week 6 were randomly assigned to continue receiving vedolizumab every 8 or 4 weeks, or to switch to placebo for up to 52 weeks. At week 52, 41.8\% of patients who continued to receive vedolizumab every 8 weeks and $44.8 \%$ of patients who continued to receive vedolizumab every 4 weeks were in clinical remission, as compared with $15.9 \%$ of patients who switched to placebo $(P<0.001) .{ }^{38}$ In GEMINI 2 (NCT00783692), an integrated study with induction and maintenance trials with vedolizumab was performed in patients with active $\mathrm{CD} .{ }^{39}$ The induction trials included 368 patients who were randomly assigned to receive vedolizumab or placebo at weeks 0 and 2 (cohort 1), and 747 patients who received open-label vedolizumab at weeks 0 and 2 (cohort 2). Disease response (based on Crohn's Disease Activity Index [CDAI]) was assessed at week 6. At week 6, $14.5 \%$ of the patients in cohort 1 and $17.7 \%$ of patients in cohort 2 were in clinical remission. In the maintenance trial, 461 patients who previously responded to vedolizumab were randomly assigned to receive placebo or vedolizumab every 8 or 4 weeks until week 52. Among patients who had a response to induction therapy, clinical remission rates at 52 weeks were $39 \%$ and $36.4 \%$ for patients assigned to vedolizumab every 8 weeks and every 4 weeks, as compared with $21.6 \%$ assigned to placebo $(P<0.001$ and $P=0.004$ for the two vedolizumab groups, respectively, vs placebo).

Given its gut-selective properties and less systemic effects, vedolizumab may have a more favorable safety profile compared to other biologics. Safety data integrated from 6 clinical trials ${ }^{39}$ with vedolizumab including more than 2800 patients demonstrated no increased risk of any infection. Vedolizumab was not associated with increased risk of serious or opportunistic infections - including sepsis, tuberculosis, Listeria meningitis, and clostridial infections-or increased malignancy when compared with placebo. Serious clostridial infections, sepsis, and tuberculosis were reported infrequently, and no cases of PML were observed. To date, only one case of PML in a vedolizumab-treated patient has been reported in the post-marketing setting. Of note, this patient had multiple contributory factors, including human immunodeficiency virus infection with a CD4 count of 300 cells $/ \mathrm{mm}^{3}$ and prior and concomitant immunosuppression. ${ }^{40}$ In the phase 3, open-label GEMINI long-term safety (LTS) study, ${ }^{41}$ which enrolled 2243 patients with IBD who received vedolizumab $300 \mathrm{mg}$ IV every 4 weeks, serious adverse events were reported for 31\% (UC) and $41 \%$ (CD) of patients over 8 years. There were no new trends for infections, malignancies, infusion-related reactions, or cases of PML in these studies. Long-term clinical response rates with vedolizumab maintenance therapy were $33 \%$ and $28 \%$ for UC and CD, respectively. In the VARSITY clinical trial comparing vedolizumab versus adalimumab in moderate to severe UC, vedolizumab was found to be superior to adalimumab for the outcome of clinical remission and endoscopic response, but not corticosteroid-free clinical remission. ${ }^{42}$ In the multicenter cohort study nested in the OBSERV-IBD cohort consisting of 294 patients with IBD on vedolizumab, $34(13.8 \%)$ patients, without any extraintestinal manifestation at baseline, developed incident cases of inflammatory arthralgia/arthritis. ${ }^{43}$

In recent years, there has been an increasing emphasis on identifying clinical and biological markers that accurately predict patient response to certain IBD treatments. ${ }^{44}$ This is particularly important for tailoring the treatment regimen to each patient. Several studies have evaluated utility of prediction models for response to vedolizumab therapy. For both UC and CD patients, baseline disease activity was identified as an independent predictor of vedolizumab response. Specifically, the GEMINI trials $^{38,39}$ found that patients with a $<9$ baseline Mayo score and $\leq 330$ CDAI score demonstrated higher rates of achieving clinical remission (at weeks 6 and 54) than the placebo group. Subsequent cohort studies have shown that patients with higher disease activity scores are associated with lower rates of clinical remission. ${ }^{45,46}$ High baseline levels of systemic and intestinal inflammation are associated with lower rates of remission and clinical response to vedolizumab. Elevated $\mathrm{C}$-reactive protein (CRP) was associated with lower rates of clinical response or remission at week 14 of vedolizumab treatment. ${ }^{45,46}$ Conversely, lower baseline CRP levels were associated with higher rates of clinical remission at week 54. Initial clinical response to vedolizumab is a predictor of longterm treatment response; those who demonstrate early responses to vedolizumab treatment have higher rates of also achieving long-term responses-specifically, steroidfree clinical remission. ${ }^{45,46}$ Prior anti-TNF failure is a recognized risk factor for response to vedolizumab. The GEMINI trials found that anti-TNF-naïve UC and $\mathrm{CD}$ patients tend to have higher rates of remission with vedolizumab than those who with prior anti-TNF exposure. $^{39,40}$ Clinical decision support tools to predict response to vedolizumab therapy have been developed and validated. A model including absence of exposure to anti-TNF, disease duration of 2 years or more, baseline 
endoscopic score, and baseline albumin concentration predicted corticosteroid-free remission during vedolizumab therapy in patients with $\mathrm{UC} .^{47}$ A similar model to predict vedolizumab response in $\mathrm{CD}$ was developed by incorporating the absence of previous treatment with an anti-TNF, absence of prior bowel surgery, absence of prior fistulizing disease, baseline level of albumin, and baseline concentration of CRP. ${ }^{48}$

Immune, genetic, and microbial biomarkers to predict vedolizumab response in IBD have also been explored. A prior study ${ }^{49}$ demonstrated that serum cytokines IL-6 and IL-8 could predict clinical response to therapy after 12 months of vedolizumab. A prior study ${ }^{50}$ demonstrated that baseline plasma levels of $\alpha 4 \beta 7$ on CD4+ T cells were higher in patients with IBD who responded to vedolizumab versus non-responders. In the same study, changes in integrin expression were not associated with $\mathrm{CD}$ response to vedolizumab. In patients with UC, increasing $\alpha 4 \beta 7$ levels with vedolizumab was associated with favorable clinical outcomes, whereas increasing $\alpha 4 \beta 1$ and $\alpha \mathrm{E} \beta 7$ correlated with negative outcomes. In a study that performed immunophenotyping of peripheral and mucosal immune cells in IBD patients on vedolizumab, the authors found that vedolizumab was not associated with abundance or phenotype of $\mathrm{T}$ cells but rather with significant changes in macrophage populations. ${ }^{51}$ Another study ${ }^{52}$ demonstrated that ileal biopsies from patients with $\mathrm{CD}$ with intestinal epithelial death caused by innate immunedriven pyroptosis predicted endoscopic improvement and clinical response to vedolizumab. A study using RNA-seq analysis of colon biopsies from patients with $\mathrm{IBD}^{53}$ demonstrated that baseline expression levels of four genes (PIWIL1, MAATS1, RGS13, and DCHS2) predicted endoscopic remission after vedolizumab, but not anti-TNF treatment. In this same study, ${ }^{53}$ monocytes, M1 macrophages, CD4 memory $\mathrm{T}$ cells, and $\mathrm{T}$ regulatory cells were enriched in vedolizumab non-responders, whereas naïve $\mathrm{B}$ cells were enriched in vedolizumab responders. The role of the gut microbiome in response to vedolizumab in IBD has also been evaluated. One study ${ }^{54}$ assessed disease activity and stool metagenomes at baseline, 14, 30, and 54 weeks; CD patients with greater community $\alpha$ diversity and higher baseline levels of Roseburia inulinivorans and Burkholderiales species were more likely to be in clinical remission 14 weeks after vedolizumab initiation. The authors hypothesized that greater baseline diversity includes anti-inflammatory microbes and metabolites that promote treatment response. Additionally, 13 biological pathways - citrulline, isoleucine, arginine, and polyamine BCAA synthesis pathways - were significantly enhanced in $\mathrm{CD}$ patients who achieved remission. ${ }^{54}$ Finally, a recent study identified low vitamin D status to predict vedolizumab primary non-response during induction and 1-year failure in patients with IBD. ${ }^{55}$ Using single-cell immunophenotyping of peripheral and intestinal immune cells, the authors demonstrated a potential link between serum vitamin $\mathrm{D}$ levels and expression of $\alpha 4 \beta 7$ on immune cells.

\section{Anti-Integrin Therapy in Clinical Trials: Etrolizumab (Anti- $\beta 7$ )}

Etrolizumab is a monoclonal antibody that blocks cellular adhesion by binding the $\beta 7$ subunit of both $\alpha 4 \beta 7$ and $\alpha E \beta 7$ integrins. The $\alpha 4 \beta 7$ and $\alpha E \beta 7$ integrins function as trafficking molecules that localize leukocytes to inflammatory sites in the gut. ${ }^{56}$ Etrolizumab binds $\alpha 4 \beta 7$ and prevents binding to MAdCAM-1, a receptor that is highly upregulated in the intestinal venules of patients with $\mathrm{UC}$ and $\mathrm{CD}$. Etrolizumab also binds $\alpha \mathrm{E} \beta 7$ and prevents interaction with E-cadherin found on intestinal mucosal cells. The $\alpha E \beta 7$-expressing lymphocytes residing in the gut participate in the inflammatory responses observed in IBD. ${ }^{57}$ Thus, the inhibition of $\alpha E \beta 7$ results in decreased inflammation and increased retention of leukocytes within the intraepithelial space of the intestinal mucosa.

Etrolizumab was evaluated for safety and tolerability in a phase 1 study involving patients with moderate to severe UC. The patients received etrolizumab either as a single dose $(0.3-10 \mathrm{mg} / \mathrm{kg})$ or as three doses $(0.5-4 \mathrm{mg} / \mathrm{kg})$ administered every four weeks. The drug was given either intravenously or subcutaneously. There were no significant dose-limiting toxicities, infusion, or injection site reactions in either single or multiple dose groups. ${ }^{58} \mathrm{~A}$ randomized, placebo-controlled phase 2 study was implemented to determine the drug's efficacy by enrolling patients with moderate to severe UC in three dosage groups, consisting of (i) etrolizumab $100 \mathrm{mg}$ (nominal) administered every four weeks; (ii) etrolizumab $420 \mathrm{mg}$ at week 0 (loading dose) followed by $300 \mathrm{mg}$ (nominal) at weeks 2, 4, and 8 ; and (iii) placebo. Patients in the etrolizumab groups were more likely to experience clinical remission at week ten compared to the placebo group. Most patients in clinical remission came from the etrolizumab $100 \mathrm{mg}$ group ( $n=39$ ), with $21 \%$ achieving remission, followed by $10 \%$ in the $300 \mathrm{mg}$ LD cohort $(n=39)$, compared with $0 \%$ in the 
placebo cohort $(n=41) .{ }^{59}$ Currently, there are several phase 3 clinical trials evaluating etrolizumab in IBD (UC: HIBISCUS I and II, GARDENIA, LAUREL, HICKORY; CD: BERGAMOT) ${ }^{60}$ and two open-label extension trials (UC: COTTONWOOD; CD: JUNIPER). In a recent update from phase 3 studies of etrolizumab in patients with moderate to severe UC, etrolizumab effectively induced remission compared to placebo but did not meet its primary endpoint as maintenance therapy. There were no major safety issues in any of the phase 3 studies reported to date. ${ }^{61}$

Concurrent studies evaluating biomarkers for predicting response to etrolizumab in patients with IBD have also been conducted. In a retrospective analysis of data collected from UC patients who participated in a phase 2 placebo-controlled trial of etrolizumab, ${ }^{57}$ gene expression levels of GZMA and ITGAE from colon biopsies identified patients who were most likely to benefit from etrolizumab. Furthermore, expression levels decreased with etrolizumab administration in patients with high baseline expression. In a recent study, ${ }^{62} \mathrm{CD}$ patients experienced a reduction in inflammatory genes and cytotoxic intraepithelial lymphocyte (IEL) gene signatures following etrolizumab therapy.

\section{Other Anti-Integrin Therapies Under Investigation}

As selective targeting of gut-tropic cells has been established as a validated approach to the treatment of IBD with reduced extra-intestinal systemic effects, numerous additional anti-integrin therapies are in development. Owing to their combinatorial tissue selectively and established mechanistic understanding, much focus is on the blockade of integrins and their ligands. Considerable efforts are also directed towards other pathways of cellular trafficking, namely the sphingosine-1-phosphate receptor, as well as G-protein coupled receptors (GPCRs) and C-C chemokine receptors (CCRs) in earlier phases of development. These ongoing programs include monoclonal antibodies, as well as additional pharmaceutical modalities, such as small molecules and peptides that have unique pharmacokinetic (PK) and pharmacodynamic (PD) properties.

\section{Abrilumab (AMG I8I/MEDI 7 I 83)}

Abrilumab is a human anti- $\alpha 4 \beta 7 \mathrm{IgG} 2 \mathrm{mAb}$ with favorable PK and PD properties amenable to subcutaneous administration. ${ }^{63-65}$ Phase $2 \mathrm{~b}$ trials of abrilumab were completed for both $\mathrm{CD}$ and UC. The phase $2 \mathrm{~b}$ placebocontrolled RCT for CD included 249 patients with inadequate or lost response to previous therapies, who were dosed with $21 \mathrm{mg}, 70 \mathrm{mg}$, or $210 \mathrm{mg}$ on day 1, week 2, week 4 , and every 4 weeks for 24 weeks, with a primary endpoint of remission at week 12 and CDAI at weeks 8 and $12 .{ }^{66}$ Although the primary endpoints were not met, treatment groups had higher rates of remission at week 12, safety measures were balanced between treatment and placebo groups, and $\alpha 4 \beta 7$ receptor occupancy was sustained. A phase 2 trial with $45 \mathrm{UC}$ patients demonstrated improved remission and safety for abrilumab compared to placebo. ${ }^{67} \mathrm{~A}$ larger phase $2 \mathrm{~b}$ trial for UC included 354 patients with inadequate or lost response to previous therapies, who were dosed with $21 \mathrm{mg}, 70 \mathrm{mg}$, or $210 \mathrm{mg}$ on day 1 , week 2 , week 4 , and every 4 weeks, with a primary endpoint of remission at week $8 .{ }^{68}$ There were significantly higher rates of remission in the two higherdose treatment groups at 8 weeks compared to placebo, and there were no cases of PML or deaths in the trial. To date, phase 3 trials for abrilumab have not been registered.

\section{PN-943}

$\mathrm{PN}-943$ is an orally administered and gut-restricted $\alpha 4 \beta 7$ antagonist peptide taking advantage of a topical route of administration. ${ }^{69} \mathrm{PN}-943$ is more effective for induction of remission in UC than PTG-100, an earlier-generation orally administered and gut-restricted $\alpha 4 \beta 7$ antagonist cysteine knot peptide developed by the same company that preferentially binds activated $\alpha 4 \beta 7$ on $\mathrm{T}$ cells. ${ }^{70,71}$ A phase 2 trial of PTG-100 was terminated, and a phase 2 trial of PN-943 is underway to evaluate the effects of $150 \mathrm{mg}, 450 \mathrm{mg}$, or placebo twice daily on 12- and 52week remission in moderate and severe UC (NCT04504383).

\section{Carotegrast Methyl (AJM300)}

Carotegrast methyl is an orally active small molecule inhibitor of $\alpha 4$, with demonstrated activity against $\alpha 4 \beta 7$ and $\alpha 4 \beta 1{ }^{72}$ The molecule is dosed thrice daily and characterized by generally mild adverse effects. ${ }^{73}$ A phase 2 a placebocontrolled RCT in 102 patients with moderately active UC who had inadequate or lost response to previous therapies demonstrated significantly higher rates of clinical remission and mucosal healing at 8 weeks in the treatment compared to placebo group, as well as no serious adverse events, including PML. ${ }^{74} \mathrm{~A}$ smaller trial in $\mathrm{CD}$ using significantly lower doses showed no significant difference in CDAI at week 4 
for carotegrast methyl compared to placebo, although the results were published in abstract form only. ${ }^{75} \mathrm{~A}$ phase 3 trial in UC is ongoing (NCT03531892).

\section{Ontamalimab (PF-00547659 or SHP647)}

Ontamalimab is a subcutaneously administered fully human $\operatorname{IgG} 2 \kappa$ anti-MAdCAM- $1 \mathrm{mAb}$ that blocks $\alpha 4 \beta 7$ binding to the MAdCAM-1 ligand, ${ }^{76}$ with no observed effects on cerebrospinal fluid lymphocytes. ${ }^{77}$ The OPERA phase 2 placebo-controlled RCT in patients with active moderate to severe CD enrolled 265 patients with inadequate or lost response to previous therapies. ${ }^{78}$ The primary endpoint was reduction in CDAI at week 8 or 12 , which was not significantly different between treatment and placebo; however, the remission rate was higher amongst patients with a higher baseline CRP. The TURANDOT phase 2 placebo-controlled RCT in active UC enrolled 357 patients with inadequate or lost response to previous therapies, who were administered one of four different treatment doses or placebo every 4 weeks. ${ }^{79}$ The primary endpoint was remission at week 12, which was significantly higher in 3 of 4 treatment groups compared to placebo. Adverse effects were similar across all groups. An open-label dose escalation extension of the TURANDOT trial demonstrated acceptable drug tolerance, with $36.1 \%$ of patients experiencing drug-related adverse events (AEs), with the most common serious $\mathrm{AE}$ being worsening or ongoing $\mathrm{UC}$, followed by serious infection. ${ }^{80}$ Ontamalimab was originally developed by Pfizer, which licensed it out to Shire back in 2016. Takeda later acquired Shire in 2019. At this time, it appears Ontamalimab will not be developed further and is unlikely enter the IBD market as Takeda announced in May 2020 that clinical trials will not restarted after disruptions caused by the COVID-19 pandemic. ${ }^{81}$

\section{Conclusions and Future Directions}

Antibody blockade of leukocyte trafficking to the gastrointestinal tract with anti-integrin therapy (anti- $\alpha 4 \beta 7$ ) is effective for patients with IBD and is associated with favorable safety profiles. Interestingly, biomarker studies suggest that the therapeutic efficacy of vedolizumab may be mediated by more non-T-cell pathways (eg, macrophages, B cells). The clinical benefit of additional blockade of cellular retention via targeting $\alpha E \beta 7$ with etrolizumab remains to be determined. Future studies are needed to further understand the exact mechanisms of antiintegrin therapies in IBD and whether novel anti-integrin pathway approaches (small molecule inhibitors of integrins, inhibition of cell adhesions molecules such as MAdCAM-1, etc.) have a place in the therapeutic armamentarium against IBD.

\section{Author Contributions}

All authors made a significant contribution to the work reported, whether that is in the conception, study design, execution, acquisition of data, analysis and interpretation, or in all these areas; took part in drafting, revising or critically reviewing the article; gave final approval of the version to be published; have agreed on the journal to which the article has been submitted; and agree to be accountable for all aspects of the work.

\section{Funding}

JG is supported by a Chan Zuckerberg Biohub Physician Scientist Scholar Award, NIH NIDDK LRP Award (L30 DK126220), and a Doris Duke Physician Scientist Fellowship Award (Grant \#2021091).

\section{Disclosure}

The authors have no conflicts of interests or financial disclosures relevant to this work.

\section{References}

1. de Souza HSP, Fiocchi C, Iliopoulos D. The IBD interactome: an integrated view of aetiology, pathogenesis and therapy. Nat Rev Gastroenterol Hepatol. 2017;14(12):739-749. doi:10.1038/ nrgastro.2017.110

2. Actis GC, Pellicano R, Fagoonee S, Ribaldone DG. History of inflammatory bowel diseases. J Clin Med. 2019;8(11):1970. doi:10.3390/ jcm8111970

3. Ng SC, Shi HY, Hamidi N, et al. Worldwide incidence and prevalence of inflammatory bowel disease in the 21 st century: a systematic review of population-based studies. Lancet. 2017;390(10114):2769-2778. doi:10.1016/S0140-6736(17)32448-0

4. Click B, Ramos Rivers C, Koutroubakis IE, et al. Demographic and clinical predictors of high healthcare use in patients with inflammatory bowel disease. Inflamm Bowel Dis. 2016;22(6):1442-1449. doi:10.1097/MIB.0000000000000763

5. Paramsothy S, Rosenstein AK, Mehandru S, Colombel JF. The current state of the art for biological therapies and new small molecules in inflammatory bowel disease. Mucosal Immunol. 2018;11 (6):1558-1570. doi:10.1038/s41385-018-0050-3

6. Hynes RO. Integrins: a family of cell surface receptors. Cell. 1987;48 (4):549-554. doi:10.1016/0092-8674(87)90233-9

7. Kinashi T. Intracellular signalling controlling integrin activation in lymphocytes. Nat Rev Immunol. 2005;5(7):546-559. doi:10.1038/ nri1646 
8. Shattil SJ, Kim C, Ginsberg MH. The final steps of integrin activation: the end game. Nat Rev Mol Cell Biol. 2010;11(4):288-300. doi: $10.1038 / \mathrm{nrm} 2871$

9. Habtezion A, Nguyen LP, Hadeiba H, Butcher EC. Leukocyte trafficking to the small intestine and colon. Gastroenterology. 2016;150 (2):340-354. doi:10.1053/j.gastro.2015.10.046

10. Song G, Yang Y, Liu JH, et al. An atomic resolution view of ICAM recognition in a complex between the binding domains of ICAM-3 and integrin $\alpha$ L $\beta 2$. Proc Natl Acad Sci. 2005;102(9):3366-3371. doi:10.1073/pnas.0500200102

11. Hemler ME, Huang C, Takada Y, Schwarz L, Strominger JL, Clabby ML. Characterization of the cell surface heterodimer VLA-4 and related peptides. J Biol Chem. 1987;262(24):11478-11485. doi:10.1016/S0021-9258(18)60831-2

12. Erle DJ, Briskin MJ, Butcher EC, Garcia-Pardo A, Lazarovits AI, Tidswell M. Expression and function of the MAdCAM-1 receptor, integrin alpha 4 beta 7, on human leukocytes. J Immunol. 1994;153 (2):517-528.

13. Rott LS, Briskin MJ, Andrew DP, Berg EL, Butcher EC. A fundamental subdivision of circulating lymphocytes defined by adhesion to mucosal addressin cell adhesion molecule-1. Comparison with vascular cell adhesion molecule-1 and correlation with beta 7 integrins and memory differentiation. J Immunol. 1996;156(10):3727-3736.

14. Wong MT, Ong DEH, Lim FSH, et al. A high-dimensional atlas of human $\mathrm{T}$ cell diversity reveals tissue-specific trafficking and cytokine signatures. Immunity. 2016;45(2):442-456. doi:10.1016/j. immuni.2016.07.007

15. Cepek KL, Shaw SK, Parker CM, et al. Adhesion between epithelial cells and T lymphocytes mediated by E-cadherin and the $\alpha \mathrm{E} \beta 7$ integrin. Nature. 1994;372(6502):190-193. doi:10.1038/372190a0

16. Cepek KL, Parker CM, Madara JL, Brenner MB. Integrin alpha $\mathrm{E}$ beta 7 mediates adhesion of $\mathrm{T}$ lymphocytes to epithelial cells. J Immunol. 1993;150(8):3459-3470.

17. De Lange KM, Moutsianas L, Lee JC, et al. Genome-wide association study implicates immune activation of multiple integrin genes in inflammatory bowel disease. Nat Genet. 2017;49(2):256-261. doi:10.1038/ng.3760

18. Meenan J, Spaans J, Grool TA, Pals ST, Tytgat GN, Van Deventer SJ. Altered expression of alpha 4 beta 7 , a gut homing integrin, by circulating and mucosal $\mathrm{T}$ cells in colonic mucosal inflammation. Gut. 1997;40(2):241-246. doi:10.1136/gut.40.2.241

19. Rubin SJS, Bai L, Haileselassie Y, et al. Mass cytometry reveals systemic and local immune signatures that distinguish inflammatory bowel diseases. Nat Commun. 2019;10(1):2686. doi:10.1038/s41467019-10387-7

20. Soriano A, Salas A, Salas A, et al. VCAM-1, but not ICAM-1 or MAdCAM-1, immunoblockade ameliorates DSS-induced colitis in mice. Lab Investigation. 2000;80(10):1541-1551. doi:10.1038/ labinvest.3780164

21. Picarella D, Hurlbut P, Rottman J, Shi X, Butcher E, Ringler DJ. Monoclonal antibodies specific for beta 7 integrin and mucosal addressin cell adhesion molecule-1 (MAdCAM-1) reduce inflammation in the colon of scid mice reconstituted with CD45RBhigh CD4+ T cells. J Immunol. 1997;158(5):2099-2106.

22. Podolsky DK, Lobb R, King N, et al. Attenuation of colitis in the cotton-top tamarin by anti-alpha 4 integrin monoclonal antibody. J Clin Invest. 1993;92(1):372-380. doi:10.1172/JCI116575

23. Rudick RA, Sandrock A. Natalizumab: alpha 4-integrin antagonist selective adhesion molecule inhibitors for MS. Expert Rev Neurother. 2004;4(4):571-580. doi:10.1586/14737175.4.4.571

24. Gordon FH, Lai CW, Hamilton MI, et al. A randomized placebocontrolled trial of a humanized monoclonal antibody to alpha4 integrin in active Crohn's disease. Gastroenterology. 2001;121 (2):268-274. doi:10.1053/gast.2001.26260
25. Ghosh S, Goldin E, Gordon FH, et al. Natalizumab for active Crohn's disease. $N$ Engl $J$ Med. 2003;348(1):24-32. doi:10.1056/ NEJMoa020732

26. Sandborn WJ, Colombel JF, Enns R, et al. Natalizumab induction and maintenance therapy for Crohn's disease. $N$ Engl J Med. 2005;353 (18):1912-1925. doi:10.1056/NEJMoa043335

27. Sakuraba A, Keyashian K, Correia C, et al. Natalizumab in Crohn's disease: results from a US tertiary inflammatory bowel disease center. Inflamm Bowel Dis. 2013;19(3):621-626. doi:10.1097/ MIB.0b013e31827eea78

28. Kane SV, Horst S, Sandborn WJ, et al. Natalizumab for moderate to severe Crohn's disease in clinical practice: the Mayo Clinic Rochester experience. Inflamm Bowel Dis. 2012;18(12):2203-2208. doi:10.1002/ibd.22943

29. Chen CH, Kularatna G, Stone CD, Gutierrez AM, Dassopoulos T. Clinical experience of natalizumab in Crohn's disease patients in a restricted distribution program. Ann Gastroenterol. 2013;26 (3):189-190.

30. Berger JR, Koralnik IJ. Progressive multifocal leukoencephalopathy and natalizumab-unforeseen consequences. $N$ Engl J Med. 2005;353 (4):414-416. doi:10.1056/NEJMe058122

31. Van Assche G, Van Ranst M, Sciot R, et al. Progressive multifocal leukoencephalopathy after natalizumab therapy for Crohn's disease. $N$ Engl J Med. 2005;353(4):362-368. doi:10.1056/NEJMoa051586

32. Padgett BL, Walker DL, ZuRhein GM, Eckroade RJ, Dessel BH. Cultivation of papova-like virus from human brain with progressive multifocal leucoencephalopathy. Lancet. 1971;1(7712):1257-1260. doi:10.1016/s0140-6736(71)91777-6

33. Bellaguarda E, Keyashian K, Pekow J, Rubin DT, Cohen RD, Sakuraba A. Prevalence of antibodies against JC virus in patients with refractory Crohn's disease and effects of natalizumab therapy. Clin Gastroenterol Hepatol. 2015;13(11):1919-1925. doi:10.1016/j. cgh.2015.05.022

34. Ho PR, Koendgen H, Campbell N, Haddock B, Richman S, Chang I. Risk of natalizumab-associated progressive multifocal leukoencephalopathy in patients with multiple sclerosis: a retrospective analysis of data from four clinical studies. Lancet Neurol. 2017;16(11):925-933. doi:10.1016/S1474-4422(17)30282-X

35. Gomollón F, Dignass A, Annese V, et al.; ECCO. 3rd european evidence-based consensus on the diagnosis and management of Crohn's disease 2016: part 1: diagnosis and medical management. J Crohns Colitis. 2017;11(1):3-25.

36. Soler D, Chapman T, Yang LL, Wyant T, Egan R, Fedyk ER. The binding specificity and selective antagonism of vedolizumab, an anti$\alpha 4 \beta 7$ integrin therapeutic antibody in development for inflammatory bowel diseases. J Pharmacol Exp Ther. 2009;330(3):864-875. doi:10.1124/jpet.109.153973

37. Feagan BG, Rutgeerts P, Sands BE, et al.; GEMINI 1 Study Group. Vedolizumab as induction and maintenance therapy for ulcerative colitis. N Engl J Med. 2013;369(8):699-710.

38. Sandborn WJ, Feagan BG, Rutgeerts P, et al. Vedolizumab as induction and maintenance therapy for Crohn's disease. $N$ Engl $J$ Med. 2013;369(8):711-721.

39. Colombel JF, Sands BE, Rutgeerts P, et al. The safety of vedolizumab for ulcerative colitis and Crohn's disease. Gut. 2017;66(5):839-851. doi:10.1136/gutjnl-2015-311079

40. ENTYVIO (vedolizumab). Entyvio ${ }^{\circledR}$ (vedolizumab) Official HCP Site; 2021. Available from: https://www.entyviohcp.com/safetyprofile. Accessed July 17, 2021.

41. Loftus EV Jr, Feagan BG, Panaccione R, et al. Long-term safety of vedolizumab for inflammatory bowel disease. Aliment Pharmacol Ther. 2020;52(8):1353-1365. doi:10.1111/apt.16060

42. Sands BE, Peyrin-Biroulet L, Loftus EV Jr, et al. Vedolizumab versus adalimumab for moderate-to-severe ulcerative colitis. $N$ Engl J Med. 2019;381(13):1215-1226. doi:10.1056/NEJMoa1905725 
43. Tadbiri S, Peyrin-Biroulet L, Serrero M, et al., GETAID OBSERV-IBD study group. Impact of vedolizumab therapy on extra-intestinal manifestations in patients with inflammatory bowel disease: a multicentre cohort study nested in the OBSERV-IBD cohort. Aliment Pharmacol Ther. 2018;47(4):485-493. doi:10.1111/apt.14419

44. Gubatan J, Levitte S, Patel A, Balabanis T, Wei MT, Sinha SR. Artificial intelligence applications in inflammatory bowel disease: emerging technologies and future directions. World J Gastroenterol. 2021;27(17):1920-1935. doi:10.3748/wjg.v27.i17.1920

45. Amiot A, Serrero M, Peyrin-Biroulet L, et al. One-year effectiveness and safety of vedolizumab therapy for inflammatory bowel disease: a prospective multicentre cohort study. Aliment Pharmacol Ther. 2017;46:310-321. doi:10.1111/apt.14167

46. Amiot A, Filippi J, Roblin X, et al. Effectiveness and safety of vedolizumab induction therapy for patients with inflammatory bowel disease. Clin Gastroenterol Hepatol. 2016;14:1593-1601. doi:10.1016/j.cgh.2016.02.016

47. Dulai PS, Singh S, Casteele NV, et al. Development and validation of clinical scoring tool to predict outcomes of treatment with vedolizumab in patients with ulcerative colitis. Clin Gastroenterol Hepatol. 2020;18(13):2952-2961. doi:10.1016/j.cgh.2020.02.010

48. Dulai PS, Boland BS, Singh S, et al. Development and validation of a scoring system to predict outcomes of vedolizumab treatment in patients with Crohn's disease. Gastroenterology. 2018;155 (3):687-695. doi:10.1053/j.gastro.2018.05.039

49. Bertani L, Caviglia GP, Antonioli L, et al. Serum Interleukin-6 and -8 as predictors of response to vedolizumab in inflammatory bowel diseases. J Clin Med. 2020;9(5):1323. doi:10.3390/jcm9051323

50. Fuchs F, Schillinger D, Atreya R, et al. Clinical response to vedolizumab in ulcerative colitis patients is associated with changes in integrin expression profiles. Front Immunol. 2017;8:764. doi:10.3389/fimmu.2017.00764

51. Zeissig S, Rosati E, Dowds CM, et al. Vedolizumab is associated with changes in innate rather than adaptive immunity in patients with inflammatory bowel disease. Gut. 2019;68(1):25-39. doi:10.1136/ gutjnl-2018-316023

52. Osterman MT, Gordon IO, Davis EM, et al. Mucosal biomarker of innate immune activation predicts response to vedolizumab in Crohn's disease. Inflamm Bowel Dis. 2019;25(Supplement_1):S41. doi:10.1093/ibd/izy393.094

53. Verstockt B, Verstockt S, Veny M, et al. Expression levels of 4 genes in colon tissue might be used to predict which patients will enter endoscopic remission after vedolizumab therapy for inflammatory bowel diseases. Clin Gastroenterol Hepatol. 2020;18(5):1142-1151. doi:10.1016/j.cgh.2019.08.030

54. Ananthakrishnan AN, Luo C, Yajnik V, et al. Gut microbiome function predicts response to anti-integrin biologic therapy in inflammatory bowel diseases. Cell Host Microbe. 2017;21(5):603-610.e3. doi:10.1016/j.chom.2017.04.010

55. Gubatan J, Rubin SJS, Bai L, et al. Vitamin D is Associated with $\alpha 4 \beta 7+$ Immunophenotypes and Predicts Vedolizumab Therapy Failure in Patients with Inflammatory Bowel Disease. J Crohns Colitis. 2021:jjab114. doi:10.1093/ecco-jcc/jjab114. Epub ahead of print. PMID: 34180967.

56. Fiorino G, Gilardi D, Danese S. The clinical potential of etrolizumab in ulcerative colitis: hypes and hopes. Therap Adv Gastroenterol. 2016;9(4):503-512. doi:10.1177/1756283X16647935

57. Tew GW, Hackney JA, Gibbons D, et al. Association between response to etrolizumab and expression of integrin $\alpha \mathrm{E}$ and Granzyme A in colon biopsies of patients with ulcerative colitis. Gastroenterology. 2016;150(2):477-87.e9.

58. Rutgeerts PJ, Fedorak RN, Hommes DW, et al. A randomised phase I study of etrolizumab (rhuMAb $\beta 7$ ) in moderate to severe ulcerative colitis. Gut. 2013;62(8):1122-1130. doi:10.1136/gutjnl-2011-301769
59. Vermeire S, O’Byrne S, Keir M, et al. Etrolizumab as induction therapy for ulcerative colitis: a randomised, controlled, phase 2 trial. Lancet. 2014;384(9940):309-318. doi:10.1016/S0140-6736(14)60661-9

60. Gene.com. Genentech: press Releases | Sunday, Aug 9, 2020; 2021. Available from: https://www.gene.com/media/press-releases/14870/ 2020-08-09/genentech-provides-update-on-phase-iii-s>. Accessed June 27, 2021.

61. Sandborn WJ, Vermeire S, Tyrrell H, et al.; Etrolizumab Global Steering Committee. Etrolizumab for the treatment of ulcerative colitis and crohn's disease: an overview of the Phase 3 clinical program. Adv Ther. 2020;37(7):3417-3431.

62. Dai B, Hackney JA, Ichikawa R, et al. Dual targeting of lymphocyte homing and retention through $\alpha 4 \beta 7$ and $\alpha E \beta 7$ inhibition in inflammatory bowel disease. Cell Rep Med.

63. Pan WJ, Hsu H, Rees WA, et al. Pharmacology of AMG 181, a human anti- $\alpha 4 \beta 7$ antibody that specifically alters trafficking of gut-homing T cells. Br J Pharmacol. 2013;169:51-68. doi:10.1111/bph.12134

64. Pan W-J, Köck K, Rees WA, et al. Clinical pharmacology of AMG 181 , a gut-specific human anti- $\alpha 4 \beta 7$ monoclonal antibody, for treating inflammatory bowel diseases. $\mathrm{Br} \quad \mathrm{J}$ Clin Pharmacol. 2014;78:1315-1333. doi:10.1111/bcp.12418

65. Lamb Ca, O’Byrne S, Keir ME, et al. Gut-selective integrin-targeted therapies for inflammatory bowel disease. J Crohn's Colitis. 2018;12: S653-S668. doi:10.1093/ecco-jcc/jjy060

66. Sandborn WJ, Cyrille M, Berner Hansen M, et al. OP035 Efficacy and safety of abrilumab (AMG 181/MEDI 7183) therapy for moderate to severe Crohn's disease. J Crohn's Colitis. 2017;11:S22-S23. doi:10.1093/ecco-jcc/jjx002.034

67. Hibi T, Motoya S, Ashida T, et al. Efficacy and safety of abrilumab, an $\alpha 4 \beta 7$ integrin inhibitor, in Japanese patients with moderate-tosevere ulcerative colitis: a phase II study. Intest Res. 2019;17:375-386. doi:10.5217/ir.2018.00141

68. Sandborn WJ, Cyrille M, Hansen MB, et al. Efficacy and safety of abrilumab in a randomized, placebo-controlled trial for moderate-tosevere ulcerative colitis. Gastroenterology. 2019;156:946-957.e18. doi:10.1053/j.gastro.2018.11.035

69. Modi NB, Cheng X, Mattheakis L, et al. Single- and multiple-dose pharmacokinetics and pharmacodynamics of PN-943, a gastrointestinal-restricted oral peptide antagonist of $\alpha 4 \beta 7$, in healthy volunteers. Clin Pharmacol Drug Dev. 2021. doi:10.1002/cpdd.946

70. Mattheakis L, Bhandari A, Bai L, et al. P-126 PTG-100, an oral peptide antagonist of integrin $\alpha 4 \beta 7$ that alters trafficking of gut homing $\mathrm{T}$ cells in preclinical animal models. Inflamm Bowel Dis. 2016;22:S48. doi:10.1097/01.MIB.0000480232.55276.b3

71. Mattheakis L, Fosser C, Saralaya R, et al. P113 Model based predictions of the PTG-100 pharmacodynamic responses in ulcerative colitis patients. J Crohn's Colitis. 2017;11:S132-S133. doi:10.1093/ecco-jcc /jjx002.239

72. Sugiura T, Kageyama S, Andou A, et al. Oral treatment with a novel small molecule alpha 4 integrin antagonist, AJM300, prevents the development of experimental colitis in mice. J Crohns Colitis. 2013;7 (11):e533-542. doi:10.1016/j.crohns.2013.03.014

73. Fukase H, Kajioka T, Oikawa I, et al. AJM300, a novel oral antagonist of $\alpha 4$-integrin, sustains an increase in circulating lymphocytes: a randomised controlled trial in healthy male subjects. $\mathrm{Br} J$ Clin Pharmacol. 2020;86:591-600. doi:10.1111/bcp.14151

74. Yoshimura N, Watanabe M, Motoya S, et al. Safety and efficacy of AJM300, an oral antagonist of $\alpha 4$ integrin, in induction therapy for patients with active ulcerative colitis. Gastroenterology. 2015;149 (7):1775-1783.e2. doi:10.1053/j.gastro.2015.08.044

75. Takazoe M, Watanabe M, Kawaguchi T, et al. S1066 oral Alpha-4 Integrin Inhibitor (AJM300) in patients with active crohn's disease a randomized, double-blind, placebo-controlled trial. Gastroenterology. 2009;136:A. doi:10.1016/S0016-5085(09)60816-7 
76. Pullen N, Molloy E, Carter D, et al. Pharmacological characterization of PF-00547659, an anti-human MAdCAM monoclonal antibody. $\mathrm{Br}$ J Pharmacol. 2009;157:281-293. doi:10.1111/j.1476-5381.2009.00137.x

77. D'Haens G, Vermeire S, Vogelsang H, et al. Effect of PF-00547659 on central nervous system immune surveillance and circulating $\beta 7+$ $\mathrm{T}$ cells in Crohn's disease: report of the TOSCA study. J Crohns Colitis. 2018;12:188-196. doi:10.1093/ecco-jcc/jjx128

78. Sandborn WJ, Lee SD, Tarabar D, et al. Phase II evaluation of antiMAdCAM antibody PF-00547659 in the treatment of Crohn's disease: report of the OPERA study. Gut. 2018;67:1824-1835. doi:10.1136/gutjnl-2016-313457

79. Vermeire S, Sandborn WJ, Danese S, et al. Anti-MAdCAM antibody (PF-00547659) for ulcerative colitis (TURANDOT): a phase 2, randomised, double-blind, placebo-controlled trial. Lancet. 2017;390:135-144. doi:10.1016/S0140-6736(17)30930-3
80. Reinisch W, Sandborn WJ, Danese S, et al. Long-term safety and efficacy of the anti-MAdCAM-1 monoclonal antibody ontamalimab (SHP647) for the treatment of ulcerative colitis: the open-label study TURANDOT II. J Crohns Colitis. 2021;15(6):938-949. doi:10.1093/ ecco-jcc/jjab023

81. FierceBiotech. Takeda culls Shire pipeline med after no one wants it; 2021. Available from: https:/www.fiercebiotech.com/biotech/takedadoesn-t-have-to-divest-shire-pipeline-med-as-no-one-wants-it-so-ittosses-it-out. Accessed August 5, 2021.

\section{Publish your work in this journal}

Clinical and Experimental Gastroenterology is an international, peerreviewed, open access, online journal publishing original research, reports, editorials, reviews and commentaries on all aspects of gastroenterology in the clinic and laboratory. This journal is indexed on American Chemical Society's Chemical Abstracts Service (CAS).
The manuscript management system is completely online and includes a very quick and fair peer-review system, which is all easy to use. Visit http://www.dovepress.com/testimonials.php to read real quotes from published authors. 